\title{
Aplicación de la Metodología Seis Sigmas para Mejorar la Calidad de la Estimación de Densidad Básica de la Madera en Acacia mearnsii
}

\author{
Pinilla, J.C. ${ }^{*} ;$ Navarrete, F. ${ }^{1}$ Luengo, K. ${ }^{1}$; González, J. ${ }^{1}$; Navarrete, M. ${ }^{1}$ y Acevedo, J. ${ }^{1}$ \\ ${ }^{1}$ Investigadores, Instituto Forestal, Sede Bio Bio. Concepción, Chile.. \\ *Autor para correspondencia: jpinilla@infor.cl
}

DOI: https://doi.org/10.52904/0718-4646.2021.470

Recibido: 20.03.21; Aceptado: 10.04.21

\section{RESUMEN}

La estimación de la densidad básica de la madera se realiza mediante el método tradicional regido por la Norma Tappi T 258 om-94, o bien, por la norma chilena NCh 176/2, las cuales son métodos destructivos, que demandan extensos tiempos, altos gastos en mano de obra y gran utilización de material.

Buscando métodos que permitan reducir esos inconvenientes se testeó la metodología Seis Sigmas, utilizando el método DMAMC (Definir, Medir, Analizar, Mejorar y Controlar), para mejorar este proceso, y estimar este parámetro en tiempos y costos más reducidos. Para esto se probó la espectroscopía NIR (Near Infrarred) calibrada para predecir la densidad de la madera en Acacia mearnsii, utilizando polvo de astillado en estado seco obtenido de tarugos.

El uso de esta tecnología fue apoyado con modelos estadísticos de regresión multivariantes de mínimos cuadrados parciales (PLS, Partial Least Squares), y se generó un modelo de calibración y un modelo de validación para el instrumento.

El objetivo de este estudio fue mejorar los tiempos del cálculo de densidad básica, parámetro físico importante para la obtención de información de la biomasa, que se emplea como un estimador del material leñoso de una especie y del rendimiento que alcanzará en el pulpaje, así como para determinar la facilidad con la que esta se puede trabajar.

Los niveles de predicción del modelo PLS fueron sobre el $80 \%$, por lo que se valida el uso de la espectroscopía NIR como herramienta para predecir la densidad básica. Además, se aprecia un aumento en los indicadores de calidad, el nivel sigma aumentó de 0,18 a 3, el Cp de 0,26 a 1.08, el Cpk de 0,23 a 1 y los defectos por millón disminuyeron en un 99.6\%. Además, utilizando la espectroscopía NIR disminuyen los costos en comparación con el método tradicional.

Palabras claves: Acacia mearnsii, Densidad básica, Espectroscopía NIR, Seis Sigmas, Metodología DMAMC.

\section{SUMMARY}

The estimation of the basic density of the wood is carried out by means of the traditional method governed by the International Standard Tappi T 258 om-94, or by the Chilean Norm NCh 176/2, which are destructive methods and demand extensive times, high labor costs and great use of material.

In the search for methods to reduce these inconveniences, the Six Sigmas methodology will be tested, using the DMAMC method (Define, Measure, Analyze, Improve and Control), to improve this process, which proposes a non-destructive methodology, which measures this parameter in shorter times and costs. For this, NIR (Near Infrared) spectroscopy calibrated to predict the density of Acacia mearnsii wood was used, using dry chipping powder obtained from dowels.

The use of this technology was supported with multivariate statistical models of partial least squares (PLS, Partial Least Squares), where a calibration model and a validation model for the instrument were generated.

The objective of this study was to improve the times of the calculation of basic density of the wood of trees of the Acacia mearnsii forest species, which is an important physical parameter for obtaining biomass information, since it is used as an estimator of the woody material of a species and the yield that it will reach in the pulping process, as well as to determine the ease with which it can be worked.

The prediction levels of the PLS model were over $80 \%$, so the use of NIR spectroscopy as a tool to predict basic density is validated. In addition, there is an increase in quality indices, the sigma level increased from 0.18 to 3 , the $\mathrm{Cp}$ from 0.26 to 1.08, the Cpk from 0.23 to 1 and the defects per million decreased by $99.6 \%$. On the other hand, using NIR spectroscopy reduces costs by $46.2 \%$ compared to the traditional method.

Keywords. Acacia mearnsii, Basic Density, Near Infrared Spectroscopy, Six Sigmas, DMAMC Methodology. 


\section{INTRODUCCIÓN}

El Instituto Forestal (INFOR) es una institución de investigación y desarrollo adscrita al Ministerio de Agricultura, que ejecuta investigaciones en el ámbito forestal. Entre los estudios de INFOR se cuentan aquellos relacionados con la caracterización de la biomasa forestal para su uso en energía, para lo cual conocer el parámetro de la densidad básica de la madera es una información relevante para los estudios de rendimiento y productividad en materia seca por hectárea de las especies forestales para la generación de energía.

Los problemas que se generan para la estimación de este parámetro se relacionan con que actualmente su medición se realiza mediante el método tradicional, regido por la Norma Internacional Tappi T 258 om-941, o mediante la Norma Chilena NCh 176/2². La diferencia que existe entre estas normas, es que en la norma Tappi se trabaja con rodelas, mientras que la norma chilena trabaja con probetas.

Para realizar la estimación de densidad básica de la madera, primero se debe determinar el volumen máximo de la madera al estado verde (lo más cercano posible al contenido de humedad máxima que corresponde al volteo del árbol), mediante desplazamiento de agua o medición directa, y luego secar las probetas a $105 \pm 2^{\circ} \mathrm{C}$ hasta obtener masa constante. A partir de ello se calcula la densidad básica dividiendo el peso seco por el volumen en verde.

Ambas metodologías son métodos destructivos y demandan tiempo para obtener una determinada cantidad de material, ya sea para extraer rodelas o probetas de árboles que se deben cosechar, con una pérdida de material y mayor gasto en mano de obra.

El estudio se centra en la utilización de herramientas de la gestión de calidad para mejorar el proceso de estimación y los tiempos para obtener la densidad básica de la madera de una especie y rodal en particular. En este caso se trabajó con árboles de Acacia mearnsii. La densidad es básica de la madera es un parámetro físico importante para la obtención de información de la biomasa, ya que se emplea como un estimador del material leñoso presente en una especie, en peso seco por unidad de superficie, cuantificándose así la biomasa para energía, pero también sus características para otros usos, como el rendimiento que alcanzará en un proceso de pulpaje o la facilidad con que esta madera se puede trabajar en procesos de corte, cepillado, moldurado, secado, impregnado y otros La densidad varía entre especies, incluso entre individuos de una misma especie, con la edad y con las condiciones de suelo, clima y de manejo silvícola a que han sido sometidos los árboles. Otros parámetros que se pueden estimar con la densidad básica son la resistencia, rigidez, dureza y calidad de los productos fabricados a partir de esta materia prima.

Entre las herramientas de gestión de calidad para facilitar este proceso, se consideró la metodología Seis Sigma $^{3}$ para poder mejorar la estimación de la densidad básica de los árboles, determinando a través de este método la característica crítica de calidad del proceso.

En este análisis y como complemento a la herramienta de gestión de la calidad, se determinó que una opción de mejora puede ser determinar si la Espectroscopía de Infrarrojo Cercano (Near Infrarred Spectroscopy, NIR) puede ser utilizada para estimar la densidad de la madera de Acacia mearnssi, utilizando polvo de astillado en estado seco obtenido de tarugos, lo que evita que el árbol sea volteado, y analizando si este método es más eficiente para su utilización en terreno. La metodología a utilizar se apoya con modelos estadísticos, como la regresión multivariables de mínimos cuadrados parciales (partial leats squares, PLS). Cada resultado obtenido a través del NIR debe ser comparado con las densidades obtenidas por el método tradicional, analizando las ventajas y desventajas de cada método, y concluyendo si la utilización de la espectroscopía NIR es efectivamente un método que mejora la calidad del proceso.

La estrategia de trabajo para esta metodología está dada por una serie de pasos, los cuales permiten llevar a cabo mejoras de los procesos de forma exitosa. A estos diferentes pasos se les conoce como DMAMC (Definir, Medir, Analizar, Mejorar y Controlar), herramienta estratégica de calidad basada en la

\footnotetext{
1 https://www.tappi.org/content/SARG/T258.pdf

2 https://ecommerce.inn.cl/nch17621986-mod.198840987

${ }^{3}$ Para mayor información se puede consultar en http://200.16.86.50/digital/33/revistas/cse/sixsigma-six.pdf
} 
estadística, que da una gran importancia a la recolección de información y a la veracidad de los datos como base para la mejora.

La actividad se enmarca en el programa de trabajo de INFOR en su línea de I\&D Biomasa Forestal y Energía y el desarrollo del Programa FIC- $\mathrm{R}^{4}$ "Fortalecimiento de la Competitividad del Sector de las Energías Renovables y de la Pyme Forestal, a través del desarrollo de herramientas de apoyo a la gestión y encadenamiento productivo para el abastecimiento sustentable de la biomasa forestal para su uso en generación de energía en la Región del Bio Bio", ejecutado por INFOR con financiamiento del Gobierno Regional de la Región del Bio Bio.

\section{OBJETIVOS}

El objetivo general es Implementar y evaluar la metodología Seis Sigmas para mejorar la calidad del proceso de la estimación de la densidad básica de la madera de árboles en pie de Acacia mearnsii, apoyado por la espectroscopía NIR.

Los objetivos específicos son:

- Evaluar la metodología Seis Sigma como herramienta para la identificación y mejora de problemas relacionados con la gestión forestal, generando indicadores del proceso que permitan realizar una comparación entre procesos y su eficiencia.

- Demostrar la importancia de la metodología DMAMC, al exigir definir de manera clara los objetivos del estudio, caracterizando y delegando las actividades necesarias para cumplir con las metas, y con ello planificar de mejor manera el proceso.

- Verificar la fiabilidad del NIR como instrumento para estimar la densidad de la madera de la especie Acacia mearnsii.

\section{ANTECEDENTES GENERALES}

El trabajo se realizó para generar respuestas frente a necesidades básicas de la gestión forestal como lo es la estimación de la densidad básica de la madera de una especie y edad determinada. Por ello, se revisaron antecedentes de la metodología Seis Sigmas y a partir de ellos la opción de utilizar la espectroscopía NIR.

\section{Antecedentes sobre la Metodología Seis Sigma}

Seis Sigma es conocido como un sistema bien estructurado, pero flexible, que permite alcanzar el éxito en los negocios, de manera sustentable. "Seis Sigma significa mejorar procesos por medio de resolver problemas" (Snee, 2001).

Se trata de una metodología que enfoca los esfuerzos en lograr altos estándares de calidad, en grupos de personas pertenecientes a una organización con líderes ampliamente capacitados que llevan a cabo proyectos de mejoramiento. Estos proyectos permiten ahorrar entre otros por concepto de:

- Un mejor uso de los recursos

- Disminución de los tiempos de ciclos

- Disminución de costos

- Aumento de rendimiento

La metodología es aplicable a una gran variedad de procesos, en los que se ven involucradas las organizaciones, abarcando desde lo netamente productivo, hasta los servicios internos y externos.

Las bases fundamentales que todo proyecto Seis Sigma debe poseer son:

- Una definición clara de los objetivos.

- Mediciones concretas y acotadas de variables asociadas a los procesos.

4 Fondo de Innovación para la Competitividad Regional FIC-R 
- Análisis estadísticos de los datos recogidos, cambios, rediseños e innovaciones.

- Un control minucioso de los cambios efectuados a los procesos intervenidos.

Seis Sigma es una implementación rigurosa, enfocada y altamente efectiva de principios y técnicas de calidad ya probados. Esta filosofía reconoce que existe una correlación directa entre el número de defectos, los costos por generar estos productos defectuosos y el nivel de satisfacción del cliente. A continuación, se describen las métricas básicas que se utilizan usualmente en Seis Sigma:

- Defecto: Cualquier error o equivocación que llega al cliente.

- Defecto por Unidad (DPU): Es el número de defectos por número de unidades producidas.

- Defectos por Millón (DPMU): Corresponde al número de defectos por millón de unidades.

- Oportunidad de Defecto (DO): Es un aspecto de una unidad donde es posible que aparezca un defecto.

- Defectos por Oportunidad (DPO): Es el número de defectos dividido por el número de oportunidades de defecto.

- Defectos por Millón de Oportunidades (DPMO): Es el índice de medida para los procesos Seis Sigma. Asume una distribución estadística normal. Se calcula como el número de defectos por unidad, dividido por el promedio de oportunidades de error en una unidad, multiplicado por un millón.

- LE: Límites de especificación. Son los límites establecidos por la empresa, basado en expectativas o experiencias.

- LC: Límites de control: Son los límites determinados por la propia variabilidad y media del proceso.

- $\quad \mathrm{C}_{\mathrm{p}}$ : Indicador de calidad que relaciona la variabilidad propia del proceso con los límites de especificación establecidos.

- $\quad C_{p k}$ : Indicador de calidad utilizado para saber si el proceso se ajusta a las tolerancias, es decir, que la media natural del proceso se encuentre centrada en relación con el valor nominal del proceso.

- Proceso Sigma: Es una medida para el desempeño de un proceso, determinado por el DPMO y una tabla de distribución normal. Entregando como ventaja que: resulta fácil de medir, fácil de entender y permite la comparación entre procesos distintos (producción v/s servicios).

Sigma $(\sigma)$ es una letra del alfabeto griego que usan los estadísticos para medir la variabilidad de un proceso. El valor de $\sigma$ indica qué frecuencia de defectos o fallos pueden ocurrir en el proceso. A más alto nivel $\sigma$, menos defectos o fallos en el proceso pueden ocurrir. De esta forma, cuando $\sigma$ aumenta la necesidad de pruebas e inspecciones disminuye, aumentando la fiabilidad del proceso, los costos de calidad disminuyen y se reducen significativamente los reprocesos. Además, el tiempo de ciclo se reduce drásticamente y la satisfacción del cliente aumenta. Un proceso con $3 \sigma$ de capacidad o tolerancia es aceptado como satisfactorio.

Existen dos aspectos importantes necesarios de considerar para poder elegir de forma adecuada qué proyectos realizar. En primer lugar, se deben identificar las restricciones de cada proceso y luego determinar el enfoque que se va a utilizar para solucionar el problema.

Toda organización tiene sus propias restricciones, las cuales se presentan de distintas formas. Cuando un proceso tiene una restricción, la secuencia de proyectos de mejoramiento a realizar se debe determinar utilizando una pauta específica. Según Goldratt y Cox (1992) estas normas son las siguientes:

- Paso 1: Identificar las restricciones del sistema.

- Paso 2: Decidir cómo explotar estas restricciones, priorizando proyectos Seis Sigma que minimicen las pérdidas. Por ejemplo, si la restricción es la demanda de mercado, se deben buscar proyectos Seis Sigma que permitan obtener un 100\% de entregas a tiempo. Si la restricción es una máquina, el enfoque debe dirigirse a disminuir el tiempo de puesta en marcha, eliminar errores y maximizar el tiempo de uso.

- Paso 3: Subordinar todo a la decisión tomada en el paso 2. Primero es necesario elegir proyectos que produzcan mejoras en las etapas inferiores del proceso (abajo en la cadena de eventos). Luego, se eligen proyectos que aseguren una oferta de recursos no defectuosos, desde etapas superiores del proceso a la restricción. 
- Paso 4: Levantar la restricción. Lo que significa que la restricción fue eliminada en los pasos 2 y 3. De no ser así, es necesario buscar proyectos que aporten recursos adicionales a la restricción. Esto puede involucrar una mayor cantidad de mano de obra o cantidad de insumos o equipamiento.

- Paso 5: Si en los pasos anteriores no se pudo eliminar la restricción, se debe volver al paso 1. Se debe pensar nuevamente el proceso como un todo. Esto significa comenzar nuevamente el ciclo.

Una vez aplicada la metodología anterior, es posible conocer de qué forma ir en busca de oportunidades de mejora, sin embargo, aún es necesario realizar análisis de costo-beneficio y se debe estimar la probabilidad de éxito de cada proyecto antes de elegir uno por sobre otro (Goldratt y Cox, 1992).

Los proyectos Seis Sigma abordan tres áreas diferentes de mejoras potenciales, calidad, costo y plazos. Las características críticas en el producto, proceso o servicio, son identificadas utilizando la notación CTx.

- Critical to Quality (CTQ): Cada unidad resultante de un proceso con características críticas en calidad, es especialmente valiosa porque, de perderse, es necesario una mayor cantidad de tiempo para reemplazarla o rehacerla. Aquellos proyectos enfocados en características críticas en calidad deben tener prioridad por sobre los otros.

- Critical to Schedule (CTS): Los proyectos que enfrentan características críticas en horarios y plazos, deben reducir los tiempos requeridos para poder producir una unidad, lo que redunda en una mayor capacidad del proceso. Este tipo de proyectos también debe tener alta prioridad.

- Critical to Cost (CTC): Los proyectos que enfrentan características críticas en costo, pueden tener ocasionalmente un efecto adverso en la calidad o el horario. Por esto, es que deben ser los que tengan la menor prioridad.

Luego de definir la característica crítica, es necesario definir bajo que método se va a trabajar. Seis Sigma posee varias opciones de metodologías, las que van a determinar cómo será abordado el proyecto desde su inicio hasta su fin. A continuación, se describe brevemente cada una de ellas:

- DMAIC: Corresponde a las siglas del inglés Define, Measure, Analyse, Improve, Control. Para definir los pasos que son necesarios llevar a cabo en todo proceso estándar Seis Sigma. Conocido en español como DMAMC.

- DMADV: Son las siglas de Define, Measure, Analyze, Design, Verify. Para definir los pasos alternativos a un proceso Seis Sigma. Corresponde a una metodología de diseño de procesos.

- DFSS: Design For Six Sigma. Define a proyectos consistentes en diseñar nuevos procesos, productos o servicios de manera que, una vez terminado, cumpla con los estándares $6 \sigma(3,4$ $\left.\mathrm{DPMO}^{5}\right)$.

El modelo utilizado en este estudio es el DMAMC, orientado al proceso del cálculo de densidad básica de la madera, centrándose en la mejora de los tiempos (CTS) con ayuda de la espectroscopia NIR.

El modelo DMAMC, como se señaló, corresponde a una estructura usada para guiar proyectos Seis Sigma, que busca el mejoramiento de procesos y que describe, en 5 partes, qué se va a hacer de forma avanzada y describe en detalle cómo se llevará a cabo. Cada etapa del ciclo DMAMC utiliza herramientas y técnicas estadísticas específicas, que sirven de apoyo para el desarrollo de la etapa en cuestión.

\section{Antecedentes de la Tecnología NIR}

La tecnología de espectroscopía NIR ha tenido gran adopción en diferentes industrias, incluyendo el sector forestal, donde existe la necesidad de una gran cantidad de estudios en análisis de parámetros físico-químicos en productos madereros y celulosa (Via, 2004). La absorbancia en algunas longitudes de onda del espectro NIR se asocia con los grupos funcionales de los polímeros que constituyen la madera, los que están a su vez, directa o indirectamente, relacionados con las propiedades químicas y/o mecánicas. Una completa revisión se puede encontrar en Tsuchikawa y Kobori (2015).

${ }^{5}$ Defectos por Millón de Oportunidades 
Esta tecnología también se está implementando en las operaciones de cosecha forestal, donde los equipos de cosecha mecanizada generalmente vienen equipados con tecnología computacional y sensores para medir las dimensiones externas de los rollizos, generalmente diámetros con corteza y longitud. El estudio de tecnologías para medir atributos relacionados a la calidad de los rollizos está progresando con diferentes niveles de éxito; ejemplos de estos son: acústica, escáner láser y óptico, rayos X, microondas, ultrasonido y espectroscopia infrarroja (NIR) (Carter et al., 2005).

Hay variados estudios en la literatura relacionados a la predicción de propiedades físicas (densidad, ángulo microfibrilar, longitud de traqueidas), mecánicas (módulo de rotura y elasticidad) y químicas (contenido de glucosa, lignina y extractivos) de la madera a partir de espectros NIR, los cuales se han llevado a cabo para coníferas y latifoliadas (Bailleres et al., 2002; Schimleck y Yazaki, 2003; Schimleck et al., 2004; Kelley et al., 2004ab; Via, 2004; Cooper et al., 2011).

Las mediciones de las propiedades de la madera dentro del fuste requieren la obtención de muestras, las cuales en el caso del NIR corresponden a astillas de aserrío que se producen durante el trozado realizado por una cosechadora o procesadora. En este sentido, es interesante recopilar información en cuanto a si las astillas de aserrío, en particular astillas en estado verde, constituyen una muestra adecuada para predecir la densidad de la madera basada en mediciones del espectro NIR.

En la actualidad, diversas empresas dedicadas al rubro forestal en Chile utilizan esta tecnología para el cálculo de la densidad básica de la madera. En la región del Bio Bio, la empresa Celulosa Arauco realiza toma de espectros a partir de viruta producida de muestras de tarugos de Pinus radiata y Eucalyptus globulus ${ }^{6}$, con errores aproximados de $30 \mathrm{~kg} / \mathrm{m}^{3}$.

La empresa forestal CMPC en los Ángeles, también utiliza la espectroscopía NIR, para diferentes usos desde $1998^{7}$, señalando que los modelos que se generan para estimar densidad básica de la madera no superan un $\mathrm{R}^{2}$ de $0,85^{8}$, con RMSEP9 de entre 20 y $30 \mathrm{~kg} / \mathrm{m}^{3}$.

Labbé et al. (2013) por su parte, utilizaron el NIR para estimar densidad básica, evaluando la variación de la densidad en el sentido longitudinal del fuste y la precisión de la espectroscopía de infrarrojo cercano (NIR) para predecir densidad y rendimiento pulpable en clones de Eucalyptus globulus, utilizando viruta, astilla y discos (rodela) en sitios de la región de La Araucanía. En dicho estudio, el valor medio de la densidad básica a la altura del DAP fue de $499 \mathrm{~kg} / \mathrm{m}^{3}$, mientras que la estimación mediante espectroscopía NIR utilizando viruta a la misma altura, fue de $498 \mathrm{~kg} / \mathrm{m}^{3}$ con un error estándar de estimación (EEE) de $28,8 \mathrm{~kg} / \mathrm{m}^{3}$ y $28,5 \mathrm{~kg} / \mathrm{m}^{3}$, respectivamente para el primer clon estudiado.

Para el segundo clon estudiado, las medias fueron de $507,1 \mathrm{~kg} / \mathrm{m}^{3}$ para la estimación mediante rodela a la altura del DAP, y de $497,2 \mathrm{~kg} / \mathrm{m}^{3}$ utilizando espectroscopía NIR, con un EE de $1,5 \mathrm{~kg} / \mathrm{m}^{3}$ y $23,2 \mathrm{~kg} / \mathrm{m}^{3}$, respectivamente.

La espectroscopia NIR, con su reducido costo de instrumentación y rápida colección espectral (con un mínimo esfuerzo requerido para la preparación de las muestras), se ajusta perfectamente a los requerimientos del análisis cuantitativo. Los beneficios y tendencias de la espectroscopia infrarroja han sido descrito por So et al. (2004), quienes señalan que "la rápida evaluación de las propiedades sólidas de la madera por medio del espectro NIR se ha convertido en un área de estudio de rápido crecimiento con amplias implicancias para la calidad de la madera y, finalmente, para el mejoramiento de los árboles, es probable que el monitoreo con NIR lleve a un aumento de la eficiencia y de las utilidades".

Sin embargo, sin la ayuda del análisis multivariado la información química del espectro NIR es muy limitada, siendo dos las técnicas de estadística multivariada más utilizadas con espectroscopia NIR, los cuadrados mínimos parciales (PLS) y el análisis de componentes principales (PCA).

\section{MATERIALES Y MÉTODO}

\footnotetext{
${ }^{6}$ Comunicación personal, Gerencia Celulosa Arauco, 2018

${ }^{7}$ Marcela San Martín, encargada de la utilización NIR CMPC Los Ángeles, comunicación personal, 2018

${ }^{8}$ Coeficiente de determinación o determinación múltiple, porcentaje de variación de la variable de respuesta que explica su relación con una o más variables predictoras

${ }^{9}$ Raíz cuadrada del cuadrado medio del error de precisión, mide la cantidad de error que hay entre dos conjuntos de datos, los valores predichos y los valores observados o conocidos
} 
En las actividades del Instituto Forestal, se contempla una línea de investigación específica sobre Biomasa Forestal y Energía, en el área de Diversificación Forestal, línea que aborda la generación, manejo y caracterización de la biomasa forestal aprovechable para una diversificación de la matriz energética; el monitoreo del mercado nacional de los combustibles derivados de la madera y estudios de las implicancias ambientales y uso sustentable de la biomasa forestal como energía, entre otros aspectos. Dicho trabajo de investigación apunta a dar respuesta a desafíos sectoriales y a contribuir a los programas de fomento al uso de la biomasa forestal como fuente de energía renovable, aprovechando y utilizando la vocación forestal de los territorios. En este accionar una de las acciones de INFOR se refiere a generar y contar con información acerca de la densidad de la madera de diversas especies forestales, ya que este valor se relaciona con la cantidad de materia seca producida por unidad de superficie, de cada especie a partir de un bosque a distintas edades y manejo forestal.

A mayor densidad, mayor será la biomasa producida en un determinado sitio y edad, lo que permite su clasificación y comparación con otras fuentes de biomasa forestal, determinando la cantidad de madera seca posible de obtener. Por lo anterior, este estudio abordó la aplicación de la metodología Seis Sigma y su evaluación en cuanto a mejorar la calidad de la estimación de densidad básica de la madera de, en este caso, Acacia mearnsii. Los resultados obtenidos serán comparados con los valores de referencia de la especie a partir de la información disponible en el país, y aquellos obtenidos donde esta especie tiene su origen, Australia, o es utilizada.

Para la obtención de las muestras de biomasa se utilizó una unidad experimental establecida por INFOR con Acacia mearnsii en el predio Santa Elvira, sector San Antonio de Cuda, comuna de Florida, región del Bio Bio. La unidad al momento del estudio contaba con 13 años, abarcando una superficie de 1 ha. Para la extracción de las muestras requeridas se realizó un censo en un $25 \%$ del área del ensayo, midiendo todos los DAP (Diámetros a la altura del pecho, 1,30 $\mathrm{m}$ de altura) de los árboles.

De cada árbol seleccionado, se obtuvo muestras de tarugos con un taladro de incremento manual, utilizando un punto ubicado a $3 \mathrm{~cm}$ sobre la extracción de cada rodela en las diferentes alturas, a 0,1 metros, 1,3 metros y a 3,2 metros de distancia hasta que el fuste tenga 5 centímetros de diámetro. Los tarugos fueron tomados desde la corteza hasta la medula del árbol, con una profundidad de entre 5 y 15 $\mathrm{cm}$ dependiendo del diámetro del árbol a lo largo de su altura. Las muestras de biomasa fueron sometidas al proceso de estimación y colecta de espectros NIR, utilizando un equipo de medición de espectroscopia NIR portátil Thermo Scientific, Modelo microPHAZIR GP®, obteniendo un determinado número de mediciones por muestra.

\section{RESULTADOS DE APLICACIÓN DE LA METODOLOGÍA SEIS SIGMA}

\section{Definir}

Luego de definir la característica crítica, se definió el método que se va a trabajar bajo la metodología Seis Sigma desde su inicio hasta su fin. Como se mencionó previamente, el modelo utilizado en este estudio fue el DMAMC, orientado al proceso del cálculo de densidad básica de la madera, centrándose en la mejora de los tiempos (CTS), planteando formalmente un problema específico de calidad identificado en el proceso de cálculo de densidad básica de la madera.

Se conformó un equipo de trabajo, designando los cargos y responzabilidades a cada integrante, definiendo como Característica Crítica de Calidad (CTQ) "el tiempo de duración del cálculo de densidad básica de la madera, en árboles de Acacia mearnsii". Es por esto que se establecen como meta "Reducir los tiempos y uso de material, para el cálculo de densidad básica de madera para la especie Acacia mearnsii, evitando el uso de métodos destructivos para obtener este valor".

Se decidió que el proceso a evaluar mediante la metodología Seis Sigma estaría dado por las actividades asociadas a los tiempos que involucran al personal de trabajo, y que sea específico de las muestras, por lo tanto, las actividades previas a la extraccion de rodelas y los tiempos dedicados al secado y saturado no serán considerados para la metodología Seis Sigma. Considerando lo recién mencionado, las actividades estudiadas son:

- Tiempo de extracción de rodela

- Tiempo de pesaje de rodela seca 
- Tiempo de medición de volumen de rodela saturada

De acuerdo con experiencias en terreno y antecedentes consultados relacionados con estimaciones de densidad básicas realizadas anteriormente en la institución, se acordó que los tiempos promedios, considerando que existen rodelas de diferentes tamaños y grosor, sería de 270 segundos, y que cada tiempo no debería demorar más de 70 segundos de este promedio, por lo que quedaron definidos los límites de especificación

$-\mathrm{LC}=270$ segundos

- LIE $=200$ segundos

- LSE = 340 segundos

Se establece la definición del problema, definición de las metas, definición del proceso, los resultados esperados y el esquema del proyecto, los cuáles se muestran en el Cuadro 1.

\section{Medir}

La medición actual para calcular la densidad básica de la madera para cualquier tipo de especie es obtenida mediante la Norma Tappi T258 om-94. Los pasos correspondientes a esta metodología son mencionados a lo largo de la etapa "Medir", en donde se cronometró cada una de las actividades mencionadas en la confirmación del proceso de la etapa "Definir".

\section{- Medición de Densidad Básica de la Madera Mediante la Norma Tappi T258 om-94}

Las muestras de biomasa fueron obtenidas desde una unidad experimental con Acacia mearnsii establecida por INFOR en el predio Santa Elvira, sector de San Antonio de Cuda, Florida, Región del Biobío. La unidad al momento del estudio contaba con 13 años, abarcando una superficie de 1 hectárea.

Cuadro 1. Cuadro Resumen Proyecto Seis Sigma

\begin{tabular}{ll}
\hline Nombre del proyecto & Disminución de los tiempos de cálculo de densidad básica de la madera. \\
\hline Institución & Instituto Forestal \\
\hline Área Involucrada & Línea de I\&D en Biomasa Forestal y Energía \\
\hline Participantes del Proyecto & Equipo técnico programa Biomasa Forestal y Energía INFOR, Sede Bio Bio \\
\hline \multirow{3}{*}{ Justificación del Proyecto } & Excesiva demora en el cálculo de la densidad básica \\
& El método tradicional es un método destructivo \\
& Gastos excesivos \\
& Pérdida de material leñoso \\
\hline Impacto en el Proceso & La entrega de avances de informes se ve condicionado por el tiempo de demora de este valor \\
& Utilización de recursos no previsto debido a contratación de ayudantes y materiales en terreno \\
\hline \multirow{3}{*}{ Oportunidades de Mejora } & Utilización de instrumentos y herramientas disponibles que podrían ser herramientas de solución \\
& Disminución en los tiempos de trabajo para los jornales encargados \\
& Utilización de la totalidad de los ensayos para los fines deseados \\
\hline Alcance del Proyecto & El alcance de este proyecto considera las actividades donde se involucra el personal para el \\
cálculo de la densidad básica de la madera. Los tiempos de las actividades que dependan de la \\
naturaleza de la muestra, no serán considerados en la aplicación de la metodología Seis \\
SigmaS. \\
El objetivo principal del proyecto es presentar una metodología no destructiva, donde se mejoren \\
los tiempos y costos para el cálculo de densidad básica de la madera.
\end{tabular}

Para la extracción de las muestras requeridas se realizó un censo en un $25 \%$ del área del ensayo. En esta etapa se numeran y miden todos los DAP (Diámetros a la altura del pecho, 1,30 m de altura) de los árboles. Este censo determinó la existencia de 334 árboles. Algunos de estos árboles presentaron uno 0 más retoños, los que al ser contabilizados generaban un total de 449 árboles. Una vez obtenida la población objetivo a través del censo realizado, se eliminaron los árboles que presentaban algún defecto. Esto correspondió a árboles de mala forma, quebrados o afectados por algún daño. Lo anterior generó una nueva población consistente en 295 árboles. Al considerar los retoños, la muestra cuenta con 391 
árboles. El diámetro promedio de esta nueva población fue de $14,4 \mathrm{~cm}$. El diámetro de los retoños en promedio alcanzo los $8,8 \mathrm{~cm}$.

Finalmente, sobre esta población se aplicó un análisis para obtener el 25\%,50\% y $75 \%$ del valor del DAP promedio observado, con el objetivo de que a partir de esos valores se realice la selección de los árboles a utilizar para la obtención de rodelas requeridas en la determinación de la densidad básica de los árboles (Bahamóndez et al., 1995). Al aplicar este procedimiento se genera el Cuadro 2.

Cuadro 2. Selección de Árboles Según DAP

\begin{tabular}{cccc}
\hline$\%$ & Árbol & $\begin{array}{c}\text { DAP Clase } \\
(\mathbf{c m})\end{array}$ & Árboles a Muestrear \\
\hline 25 & 74 & 12,2 & 20 \\
\hline 50 & 148 & 14,2 & 20 \\
\hline 75 & 221 & 16,3 & 20 \\
\hline
\end{tabular}

Lo anterior implica que los árboles donde se deberá realizar la extracción de muestras corresponderán a aquellos que cuenten en promedio con un DAP cercano a los 12,2; 14,2 y 16,3 centímetros.

Elegidos los árboles, se cortaron rodelas de entre 3 y $5 \mathrm{~cm}$ de espesor a intervalos regulares a lo largo del fuste: a 0,1 m, 1,3 $\mathrm{m}$ y cada $3,2 \mathrm{~m}$ hasta que el diámetro del fuste tenga un diámetro límite de $5 \mathrm{~cm}$, con corteza. Cada rodela fue rotulada con un código de identificación y fueron recolectadas cerca de 300 rodelas en terreno que fueron llevadas a las instalaciones del Instituto Forestal en San Pedro de la Paz, Concepción; para realizar el proceso.

El secado de las muestras demoró 7 días, y consistió en depositar las rodelas en hornos de secado Memmert $\AA$ a $103 \pm 2{ }^{\circ} \mathrm{C}$. Una vez que se obtuvo el peso seco de las rodelas, estas fueron llevadas a un salón que disponía de dos recipientes de plásticos de $44,5 \mathrm{~cm} \times 60 \mathrm{~cm} \times 40,5 \mathrm{~cm}$. Las muestras fueron depositadas en los recipientes, y posteriormente se cubrieron con agua para saturar las rodelas. Este proceso demoró15 días. En la Figura 1, se puede apreciar los distintos pasos de este proceso.

Para calcular el volumen de cada rodela, se utilizó un tarro de $20 \mathrm{~L}$, en donde se perforó a $40 \mathrm{~cm}$ de altura, y se le instaló una llave de paso, sellando bien los espacios. El tarro se llenó de agua, luego se eliminó el exceso de agua hasta quedar a nivel con la llave. Cada rodela se depositó adentro del tarro con el agua ya nivelada y con la llave de paso cerrada, esto generó un desplazamiento de agua, produciendo que el agua se moviera constantemente. Se esperó a que el agua se estabilizara y se procedió a abrir la llave de paso, para expulsar el agua sobrante, depositándola en un recipiente previamente pesado. Posteriormente se pesó el agua desplazada obteniendo su volumen. Este proceso demoró entre 3 a 7 minutos por muestra, según la naturaleza de cada muestra y el tiempo final del proceso fue de alrededor de 35 horas.

Finalmente, para el cálculo de la densidad básica de la madera, se divide el peso seco de la rodela con el volumen saturado, obtenidos en los pasos anteriores.

La media de la densidad básica fue de $712.94 \mathrm{~kg} / \mathrm{m}^{3}$ con una desviación estándar de $37.08 \mathrm{~kg} / \mathrm{m}^{3}$, en donde su valor mínimo fue de $612,15 \mathrm{~kg} / \mathrm{m}^{3}$ y máximo de $806,27 \mathrm{~kg} / \mathrm{m}^{3}$.

La literatura señala que Acacia mearnsii, es una latifoliada de densidad media, con valores que fluctúan entre los $530-598 \mathrm{~kg} / \mathrm{m}^{3}$ (densidad básica), siendo su madera dura, pero moderadamente fácil de trabajar y fácil de pulir. En estado seco y húmedo es de dureza media (Kannegiesser, 1990). La madera de Acacia mearnsii, tiene potencial para leña y carbón ya que es una madera medianamente densa que se parte fácilmente y enciende bien. Posee un peso específico de 0,70 a $0,85 \mathrm{~g} / \mathrm{cm}^{3}$ y un poder calorífico de 3.494 a $3.993 \mathrm{kCal} / \mathrm{kg}$ (Kannegiesser, 1990). Pinilla (2000) señala también que esta especie presenta una densidad básica de aproximadamente $630 \mathrm{~kg} / \mathrm{m}^{3}$ y una densidad de la madera seca al aire de entre $550 \mathrm{y}$ $800 \mathrm{~kg} / \mathrm{m}^{3}$. 
En otro estudio, Searle and Owen (2005, se calculó la densidad básica de la madera en distintas especies y en Acacia mearnsii encontraron medias de $663 \mathrm{~kg} / \mathrm{m}^{3}, 726 \mathrm{~kg} / \mathrm{m}^{3}, 651 \mathrm{~kg} / \mathrm{m}^{3}$ y $664 \mathrm{~kg} / \mathrm{m}^{3}$, según las distintas procedencias analizadas.

En Brasil, la Asociación Brasileña de Celulosa y Papel (ABTCP, 2008), indica que la densidad básica de esta especie fluctúa entre los 550 y $630 \mathrm{~kg} / \mathrm{m}^{3}$ en árboles de 7 años de edad.

\section{- Identificación de la Causa del Problema}

Se sabe que el proceso es lento, desconociéndose las causas que provocan esto. Para poder conocer estas causas, se desarrolló un diagrama de Causa-Efecto, para encontrar las causas del problema. Esto fue revisado por los miembros por los miembros del equipo y las causas fueron clasificadas en cuatro categorías: Personal, Máquinas, Método, Clima. El diagrama de causa y efecto asociado a este estudio se muestra en la Figura 2.
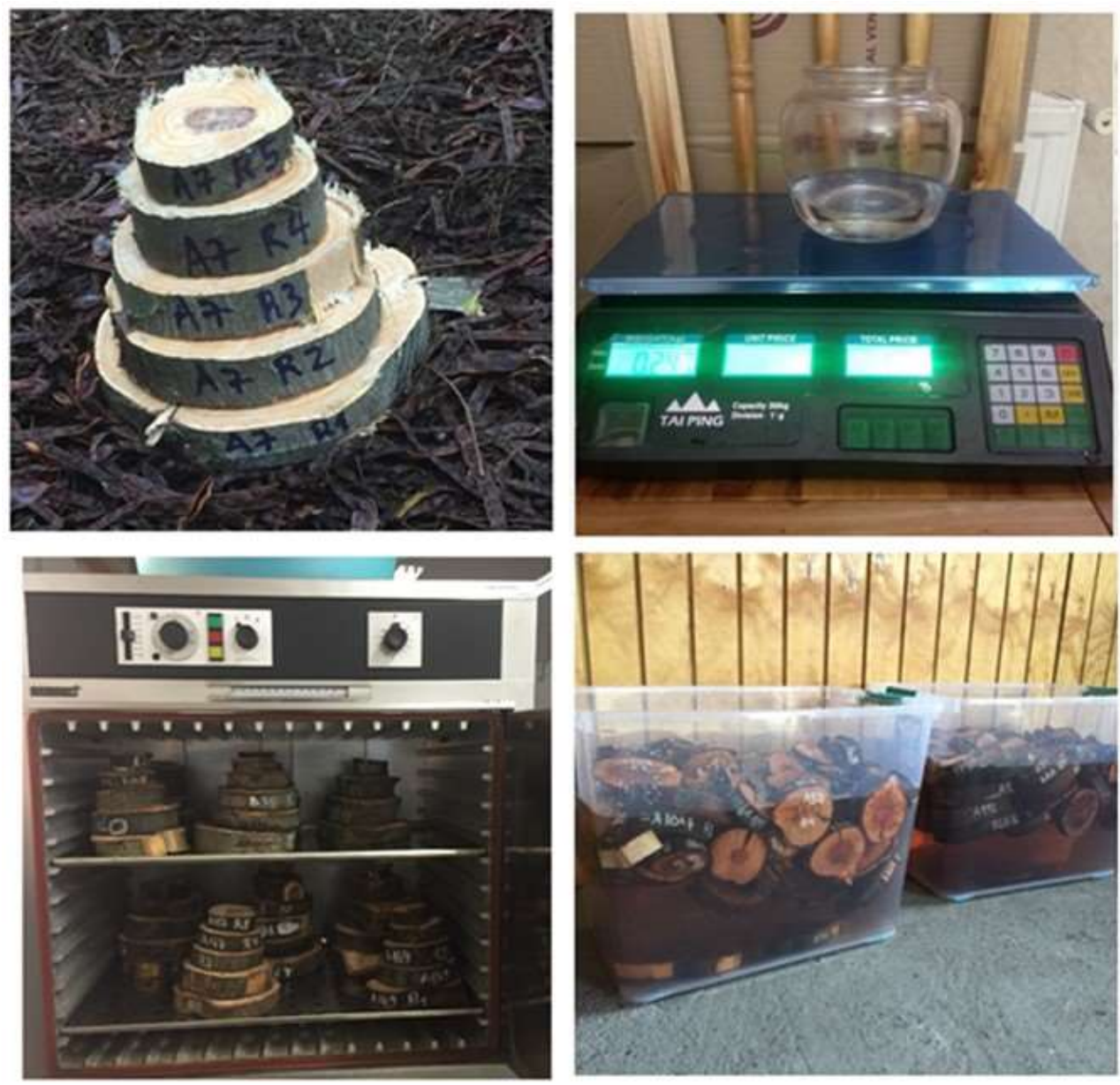

Figura 1. Proceso de Estimación de Densidad Básica de la Madera Mediante la Norma TAPPI 


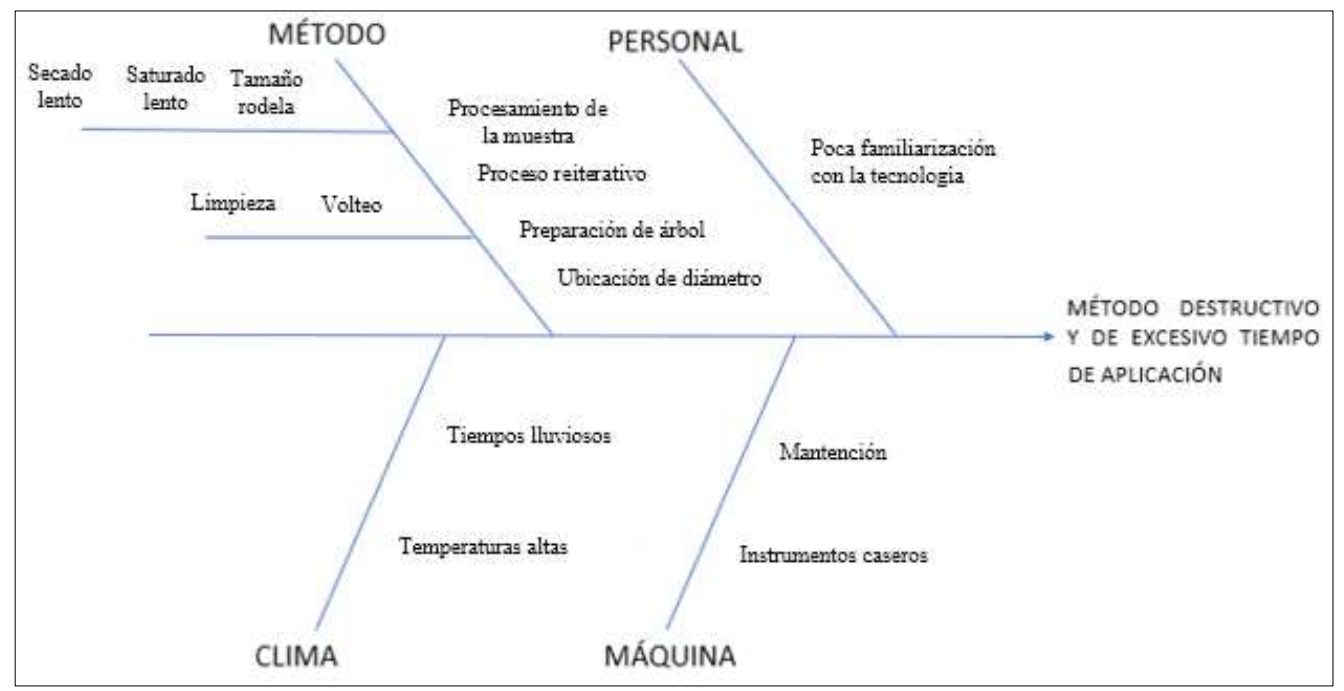

Figura 2. Diagrama Causa-Efecto de Proceso

Como se puede apreciar en el diagrama causa-efecto, se identificaron 8 causas que podrían explicar las demoras en calcular la densidad básica de la madera mediante el método tradicional. Estas causas está relacionadas con:

- Procesamiento de las muestras

- Procesos reiterativos

- Preparación de árboles

- Ubicación de diámetro de $5 \mathrm{~cm}$

- Clima en terreno (alta temperatura)

- Clima en terreno (Tiempos lluviosos)

- Poca familiarización con la metodología de trabajo

- Mantención de maquinaria.

En primera instancia se puede ver que la categoría "Método" presenta mayores causas que generan el problema, pero es necesario saber que tan importante son estas causas para el equipo de trabajo. Para esto se utiliza la Técnica de Grupo Nominal (TGN), con el fin de consensuar cuales son las causas de mayor importancia (Cuadro 3).

Cuadro 3. Técnica de Grupo Nominal

\begin{tabular}{lc}
\hline Interpretación & Puntaje \\
\hline Muy Importante & 4 \\
\hline Importante & 3 \\
\hline Poco Importante & 2 \\
\hline Nula Importancia & 1 \\
\hline
\end{tabular}

Todos los integrantes realizaron su calificación a cada una de las causas, resultados que son presentados en el Cuadro 4. 
Cuadro 4. Calificación de Importancia

\begin{tabular}{lc}
\hline Causa & Total \\
\hline Procesamiento de la muestra & 24 \\
\hline Procesos reiterativos & 22 \\
\hline Preparación de árboles & 9 \\
\hline Ubicación de diámetro de 5 cm & 8 \\
\hline Clima en terreno (alta temperatura) & 14 \\
\hline Clima en terreno (Tiempos lluviosos) & 20 \\
\hline Poca familiarización con la metodología & 11 \\
\hline Mantención & 9 \\
\hline
\end{tabular}

Con este procedimiento se determinó que las causas de mayor impacto para el equipo de trabajo son "Procesamiento de las muestras" y "Procesos reiterativos", estando este último factor asociado a la realización de actividades recurrentes y en algunos casos, tediosas.

Finalmente, con los datos obtenidos, se genera un Diagrama de Pareto (Figura 3), el cual clasifica por categoría cuales son la de mayor importancia para poder mejorar el proceso.

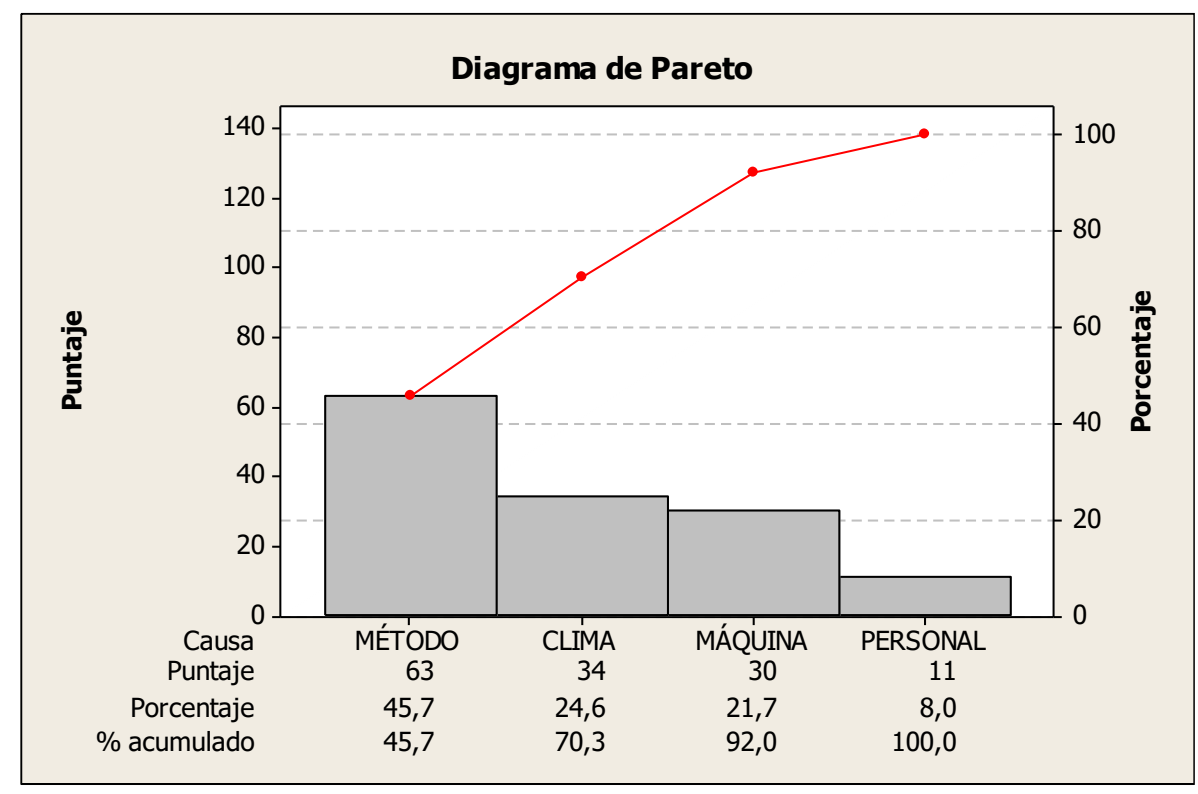

Figura 3. Clasificación Según Categoría de Importancia para Mejoras del Proceso

Se puede observar en el diagrama, que la categoría más importante para el equipo de trabajo es la del "Método de trabajo", con un 45,7\%; seguido del "Clima" con un 24,6\% y "Máquinas" con un 21,7\%; finalmente la categoría de menor importancia fue "Personal" con un $8 \%$.

A partir de la información anterior es posible en este proceso definir:

- Característica Crítica de Calidad: "Tiempo de duración del cálculo de densidad básica de la madera, en árboles de Acacia mearnsii"

- Meta: "Reducir los tiempos y uso de material, para la estimación de la densidad básica de la madera de la especie Acacia mearnsii, evitando el uso de métodos destructivos" 


\section{Analizar}

Para el análisis se seleccionaron los tiempos de 32 árboles a los cuales se le extrajeron 5 rodelas, teniendo un total de 160 muestras. La variable de observación es "tiempo", medido en segundos, las cuales fueron procesadas por el software Minitab 16.

\section{- Evaluaciones Estadísticas de Datos}

Para identificar que el proceso cumple con los criterios básicos de calidad, es necesario realizar un estudio estadístico de datos. Para esto se analizaron los datos recolectados, utilizando el software Minitab 16. En el Cuadro 5 se presenta la información más relevante.

Cuadro 5. Datos Estadísticos del Proceso Mediante la Norma Tappi

\begin{tabular}{lc}
\hline Número de muestras & 160 \\
\hline Media (segundos) & 277,91 \\
\hline Desviación Estándar (segundos) & 87,98 \\
\hline Nivel Sigma $^{10}$ & 0,18 \\
\hline
\end{tabular}

Los tiempos obtenidos mostraron una media de 277,91 segundos, 7,91 segundos más que el límite de especificación establecido por el grupo de trabajo (270 segundos). Esto refleja que la curva normal esta levemente desplazada a la derecha con respecto a los límites de especificación.

Se generan gráficos de control $\bar{X}-\mathrm{R}$, para poder evaluar la calidad del proceso. Para generar estos gráficos, a cada subgrupo se le calculó la media y el rango de sus tiempos de estimación de densidad básica, ubicando las medias en el gráfico $\bar{X}$ y los rangos en el gráfico $\mathrm{R}$. Luego, se evaluó el nivel sigma del proceso con el fin de determinar los límites de control. El nivel sigma del proceso tradicional es de 0,18 , valor excesivamente bajo, esto ocurrió debido a que los rangos de los subgrupos son muy altos y variables. Esto implica que, dentro de un subgrupo, las muestras presentan tiempos muy diferentes, en donde varias de las observaciones no están dentro de los límites de especificación. Las ecuaciones de cálculo de los límites de control son:

$$
\begin{gathered}
L I C=\hat{\mu}-Z \hat{\sigma} \\
L C=\hat{\mu} \\
L S C=\hat{\mu}+Z \hat{\sigma}
\end{gathered}
$$

Donde:

LIC es el límite inferior de control

LC es el límite central (indica que tan desplazada está la media real de la media esperada)

LSC es el límite inferior de control

$\hat{\mu}$ es la media estimada de los subgrupos

$\hat{\sigma}$ es la desviación estándar estimada de los subgrupos

$Z$ es el nivel sigma asociado al proceso

En el gráfico $\bar{X}$, los límites de control con un nivel sigma de 0,18 fueron de ${ }^{11}$ :

$$
\begin{aligned}
& - \text { LSC }=294,2 \\
& - \text { LC }=277,9 \\
& \text { - LIC }=261,7
\end{aligned}
$$

\footnotetext{
${ }^{10}$ Es una medida para el desempeño de un proceso, determinado por el DPMO y una tabla de distribución normal

${ }^{11}$ Valores obtenidos mediante el Software Minitab 16
} 
Además, en el gráfico R los límites de control con el mismo nivel sigma son los siguientes ${ }^{12}$ :

- LSC $=224,1$

$-\mathrm{LC}=210,1$

$-\mathrm{LIC}=196,0$

Mediante el gráfico de control $\bar{X}-\mathrm{R}$ (Figura 4), se pueden observar si existen o no puntos atípicos.

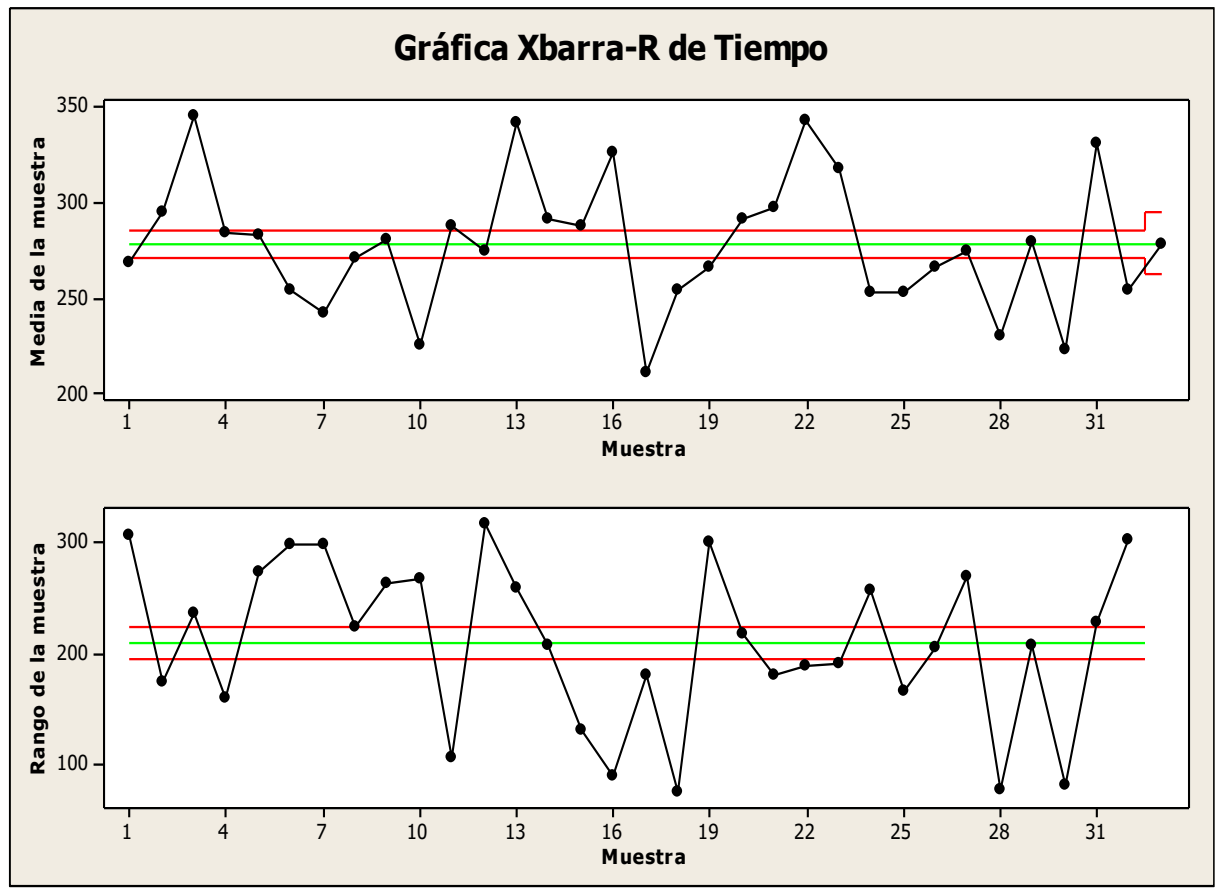

Figura 4. Gráfico de Control $\bar{X}-\mathrm{R}$

Se observa en ambos gráficos que la mayoría de los tiempos no están dentro de los límites de control, lo que confirman la alta variabilidad de las medias de las muestras, con rangos altos y variables, lo que significa una débil evaluación en términos de calidad.

Por lo tanto, debido a lo mencionado se declara que el proceso de estimación de densidad básica de la madera no se encuentra en control estadístico, por lo que se deberá generar un análisis de las causas del problema.

\section{- Análisis en la Capacidad del Proceso}

En la etapa "Definir" se estableció, mediante la reunión del grupo de trabajo, que los límites de especificación serían de 200 para el límite inferior y 340 para el límite superior.

Se realiza el análisis de capacidad del proceso, utilizando los datos recolectados, que se pueden ver en el Cuadro 6.

${ }^{12}$ Valores obtenidos mediante el Software Minitab 16 
Cuadro 6. Indicadores de Capacidad

\begin{tabular}{lc}
\hline Indicador & Valor \\
\hline $\mathrm{Cp}$ & 0,26 \\
\hline $\mathrm{Cpk}$ & 0,23 \\
\hline Nivel Sigma & 0,15 \\
\hline$\%$ defectos & 44,01 \\
\hline DPMO (Defectos por Millón de Oportunidades) & 440.059 \\
\hline
\end{tabular}

Para este caso, el valor de $\mathrm{Cp}$ es de 0,26. Al ser un valor inferior a 1, expresa que el proceso no es capaz de producir dentro de los límites de especificación preestablecidos, además, se tiene que Cpk es de 0,23, indicando que el proceso se encuentra centrado en los límites de especificación, pero con un alto número de muestras fuera de estos.

Estos resultados confirman la alta variabilidad de este proceso, por lo que se deben buscar soluciones para determinar una estabilidad de estos tiempos.

Se puede observar además que el DPMO esperado es igual a 440.059, esto quiere decir que, de un millón de observaciones, 440.059 serán defectuosas, es decir no cumplirán con los tiempos establecidos por los límites de especificación, reflejando un $44,01 \%$ de defectos.

Es indispensable entonces realizar cambios y mejoras en el proceso con el fin de optimizar los tiempos para calcular la densidad básica de la madera.

\section{- Análisis de la Causas}

Se analizó por categorías las causas identificadas en la etapa "Medir", con relación a los tiempos de demora para calcular la densidad básica de la madera. Según el Diagrama de Pareto, generado en la sección "Medir", se pudo clasificar según la importancia de las categorías, en donde el método utilizado, fue la categoría más influyente en el problema. Se decide centrar las decisiones de mejora, en esta categoría, debido a que la mayoría de las causas del problema ocurren en el método.

Se decidió utilizar la espectrometría NIR, como herramienta de mejora del proceso, ya que este instrumento ofrece versatilidad en su utilización, dado que con las mismas muestras se pueden generar modelos que no solo estime la densidad básica de la madera, sino que, además, entregue información de otros parámetros químicos, como nivel de lignina, hemicelulosa, taninos, otros.

\section{Mejorar}

En esta nueva etapa se deben desarrollar y evaluar estas oportunidades de mejora, llevando a cabo nuevas técnicas o formas más efectivas de optimización. Debido a que la opción de mejora consiste en la utilización del equipo de espectroscopía NIR, se dispuso programar y ajustar el equipo para ser utilizado en la estimación de la densidad básica de árboles en pie.

\section{- Aplicación de la Tecnología NIR para Estimación de la Densidad Básica}

De cada árbol seleccionado, se obtuvo muestras de tarugos con un taladro de incremento manual, utilizando un punto ubicado a $3 \mathrm{~cm}$ sobre la extracción de cada rodela en las diferentes alturas, a 0,1 m, $1,3 \mathrm{~m}$ y a $3,2 \mathrm{~m}$ de distancia hasta que el fuste tenga $5 \mathrm{~cm}$ de diámetro. Los tarugos fueron tomados desde la corteza hasta la medula del árbol, con una profundidad de entre 5 y $15 \mathrm{~cm}$ dependiendo del diámetro del árbol a lo largo de su altura. Se obtuvo un tarugo por cada rodela, colectando un total de 272 tarugos, los que fueron rotulados y trasladados a los laboratorios del Instituto Forestal para sus análisis posteriores.

Las muestras fueron sometidas a un proceso de secado, molido, tamizado y rotulados para sus análisis posteriores (Figura 5). Se realizó un secado de tarugos utilizando un horno Memmert ${ }^{\circledR}$ a $103 \pm 2{ }^{\circ} \mathrm{C}$ hasta 
obtener el peso constante. Los tarugos fueron molidos por un molino Wiley TE-680, obteniendo un polvo fino, el cual fue tamizado para homogenizar la granulometría de las partículas de polvo, utilizando un tamiz Prüfsieb ASTM-Nro.35 de granulometría $500 \mathrm{my}^{13}$. Posteriormente, este material se depositó en viales de vidrio para espectroscopia de aproximadamente $28 \mathrm{~cm}^{3}$ para almacenar y colectar los espectros $\mathrm{NIR}$ del polvo de astilla, las cuales nuevamente fueron sometidos a un proceso de secado, a $103 \pm 2{ }^{\circ} \mathrm{C}$, con la finalidad de extraer la humedad restante.

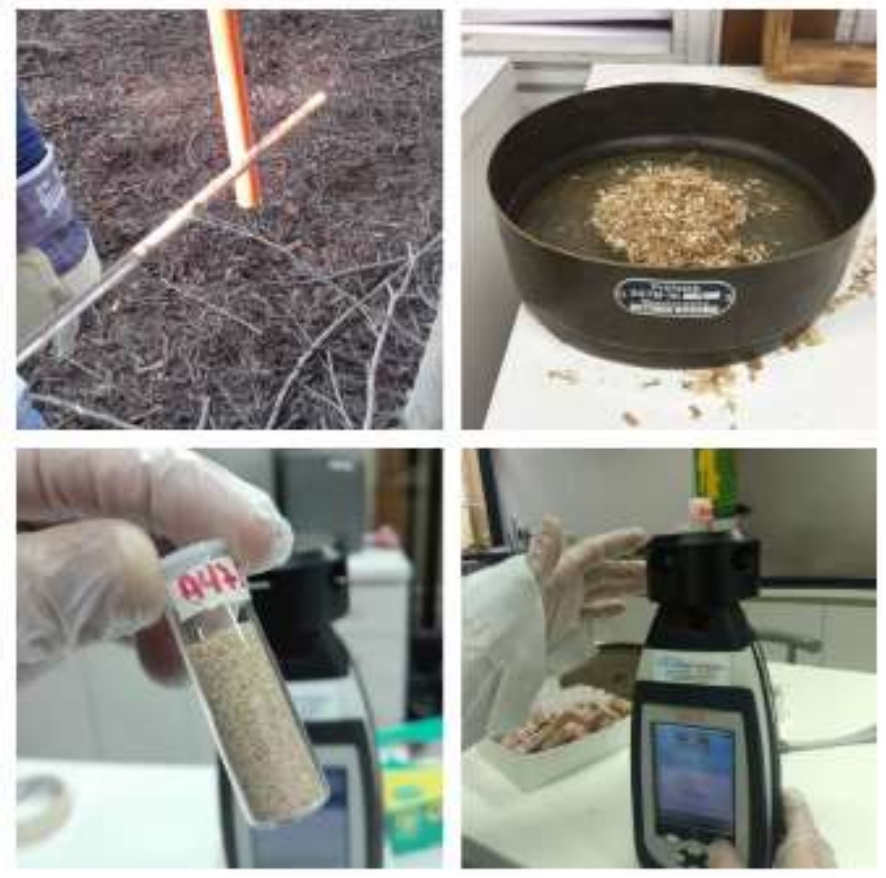

Figura 5. Obtención de Polvo de Astilla

De cada muestra, se colectaron espectros NIR, utilizando un equipo de medición de espectroscopia; NIR portátil Thermo Scientific: Modelo microPHAZIR GP®, el cual posee un rango espectral de 1.597 a $2.397 \mathrm{~nm}^{14}(6250-4166.67 / \mathrm{cm})$, con una resolución de $8 \mathrm{~nm}$, registrando la absorbancia. Cada colecta de espectros NIR, se hizo utilizando 5 mediciones por muestra, por triplicado (15 espectros por muestra), lo cual implicó tiempos de uso del escáner de 15 a 45 segundos por cada muestra.

Se utilizaron las muestras de tarugo molido y tamizado, utilizando los viales de vidrios y un adaptador del equipo para esta forma de toma de datos, considerando que se colectaron tarugos a varias alturas del árbol, se logró colectar 4.080 espectros.

La información obtenida de estas muestras se registró y almacenó en la biblioteca del equipo. Este valor permitirá, una vez obtenida la densidad básica del árbol, calculada por el método tradicional de cada muestra seleccionada, generar el primer par de datos, NIR-Densidad Básica.

El espectro obtenido en cada muestra fue promediado para proporcionar tres espectros por muestra, el que fue utilizado para predecir la densidad de la muestra (Kelley et al. 2004a).

Una vez obtenidos los datos, se genera una base de datos en el NIR, con una comparación entre los espectros obtenidos de la maquina con la densidad obtenida por el método tradicional. Al hacer esto, el NIR genera una regresión multivariada de mínimos cuadrados parciales, la cual predice, la densidad básica de la madera.

\footnotetext{
${ }^{13}$ Unidad de medida utilizada en la granulometría.

${ }^{14}$ Nanómetros o $10^{-9}$ metros
} 
De los espectros colectados, se generaron dos bases espectrales, una utilizada para la calibración de los modelos y otra para la elaboración del set de validación.

Se consideró un criterio para considerar que un espectro tenía bien asociada su densidad básica, el cual se basó principalmente en la naturaleza de las densidades obtenidas por el método tradicional.

En un árbol, la densidad básica de la madera varía según su altura, y mientras mayor sea esta menor es la densidad. Por lo tanto, para un mismo árbol si las rodelas obtenidas de alturas más altas presentan densidades más altas, los espectros asociados fueron eliminados. En caso de densidades intermitentes a lo largo de un mismo árbol, se eliminan los espectros asociados a todo el árbol.

Esta decisión fue tomada con el objetivo de cumplir con la lógica de la literatura. Cabe destacar que este criterio no es absoluto, es decir, se puede dar en la naturaleza de Acacia mearnsii casos contrarios.

Para generar estas bases de datos, de la totalidad de las muestras seleccionadas, el $75 \%$ de las muestras fue destinado a la calibración (aproximadamente 170 muestras) y el 25\% restante se destinó a la validación (47 muestras).

Con la base de calibración se genera un modelo de predicción mediante el software Thermo Scientific: Method Generator ${ }^{\circledR}$ Version4.0 R2. Este modelo es utilizado por el equipo NIR para estimar la densidad básica de la madera.

La base de predicción sirve para evaluar si el modelo realizado con la base de calibración es efectivamente bueno, para lo cual se utilizan estándares de predicción como el $R^{2}$, RMSECV ${ }^{15}$ y RMSEP para la validación de la regresión generada por el programa.

Para generar el modelo fue necesario utilizar un modelo de regresión multivariante de mínimos cuadrados parciales. Se utilizó la base de espectros de calibración en el software, cien variables de decisiones, las cuales indican la cantidad de energía absorbida por la muestra en los diferentes niveles de longitud de onda emitida por la luz del equipo.

\section{- Modelo de Calibración}

Respecto del modelo de calibración, el mejor modelo resultó ser con un suavizado de Golay de primera derivada y 13 puntos de suavización. Además, se restringieron los rangos de las curvas, en donde el rango a evaluar fue desde los 1.650-1.800 nm y 2.000-2.300 nm. Esto se hizo con el objetivo de mantener al margen la absorción del grupo funcional $\mathrm{O}-\mathrm{H}$ (hidroxilo), debido a que tiene gran relación con la humedad existente en la muestra.

La búsqueda de puntos atípicos (outler) se realizó utilizando la función por defecto del programa, la cual consiste en un coeficiente de correlación que se calcula mediante la distancia euclidiana. Esto identificó espectros mal tomados, lo cuales fueron eliminados. Una vez identificado y removido los outlers, se aplicó el proceso del modelo PLS1 ${ }^{16}$. Para esto, se calculó 20 componentes PLS ${ }^{17}$ con el método de validación cruzada Full. Este método lo que hace es retirar un espectro y generar una estimación de su densidad con los espectros restantes, esto lo hace con cada uno de sus espectros generando un modelo predictivo. Una vez se generó el modelo de prueba, se observaron puntos que presentaban puntos outler y fueron removidos del software. Finalmente, la base de calibración, luego de remover los espectros necesarios, quedó en 160 muestras, con un total de 480 espectros.

Analizando los distintos gráficos que muestra el software, se decide utilizar 6 componentes PLS, para la elaboración del modelo. Al correr el programa, se observa un $R^{2}$ de 0,813 , lo que indica que la representatividad del modelo es de un $81,3 \%$, o bien que el modelo explica en un $81, .3 \%$ los valores obtenidos. Por lo otro lado el RMSECV es de 17,54 , esto indica que el promedio de los valores absolutos de los errores en la validación cruzada son de $17,54 \mathrm{~kg} / \mathrm{m}^{3}$, donde se pudo apreciar en el software que

\footnotetext{
${ }^{15}$ Error cuadrático medio de la validación cruzada, que permite evaluar los resultados de un análisis estadístico y garantizar que son independientes de la partición entre datos

${ }_{16}$ Modelo Partial Least Squares 1, significa que el modelo trabaja con un solo vector de variables, si la situación requiere de dos o más vectores de variables, el modelo se llamaría modelo PLS2.

17 Un componente PLS, es aquel que permite mejorar el modelo, minimizando la multicolinealidad entre variables predictoras, teniendo, además, la precaución de no sobrestimar el modelo.
} 
los errores fluctuaron entre 0 y $32 \mathrm{~kg} / \mathrm{m}^{3}$. Este error es bajo en comparación con estudios mostrados en los antecedentes, donde sus RMSECV en densidad fluctúan entre 20 y $30 \mathrm{~kg} / \mathrm{m}^{3}$.

\section{- Modelo de Validación}

Mediante la utilización de la base de validación se analizó si el modelo generado es efectivamente eficiente. Para esto se cargó esta base en el software y se le implemento el modelo generado. EI RMSEP es de 18,85; con un nivel de predicción de 0,8196, lo cual es cercano al de calibración por lo que se descarta un sobreajuste o subajuste del modelo.

\section{- Modelo de Estimación de la Densidad Básica de la Madera de Acacia mearnsii}

La siguiente ecuación muestra la regresión resultante con los valores obtenidos de Thermo Scientific.

$$
\widehat{\boldsymbol{Y}}=95.601611+0.8655212 \boldsymbol{X}
$$

Siendo $\widehat{\boldsymbol{Y}}$, el vector de densidades básicas estimadas y $\mathbf{X}$ la matriz de absorción de energía de la muestra, por cada longitud de onda de la luz emitida por el equipo. A continuación, en la Figura $N^{\circ} 5$, se presentan los resultados de los modelos de calibración y validación.

Luego de calibrar el equipo NIR, el instrumento quedó preparado para medir densidades básicas de la madera, siendo necesario extraer tarugos de la misma especie, molerla para generar viruta, tamizar las muestras, secar las muestras y medir con el equipo. Al estimar las densidades a través de espectros y compararla con los valores calculados con el método tradicional, se genera el Cuadro 7, que presenta un resumen que compara los principales resultados de ambos métodos.

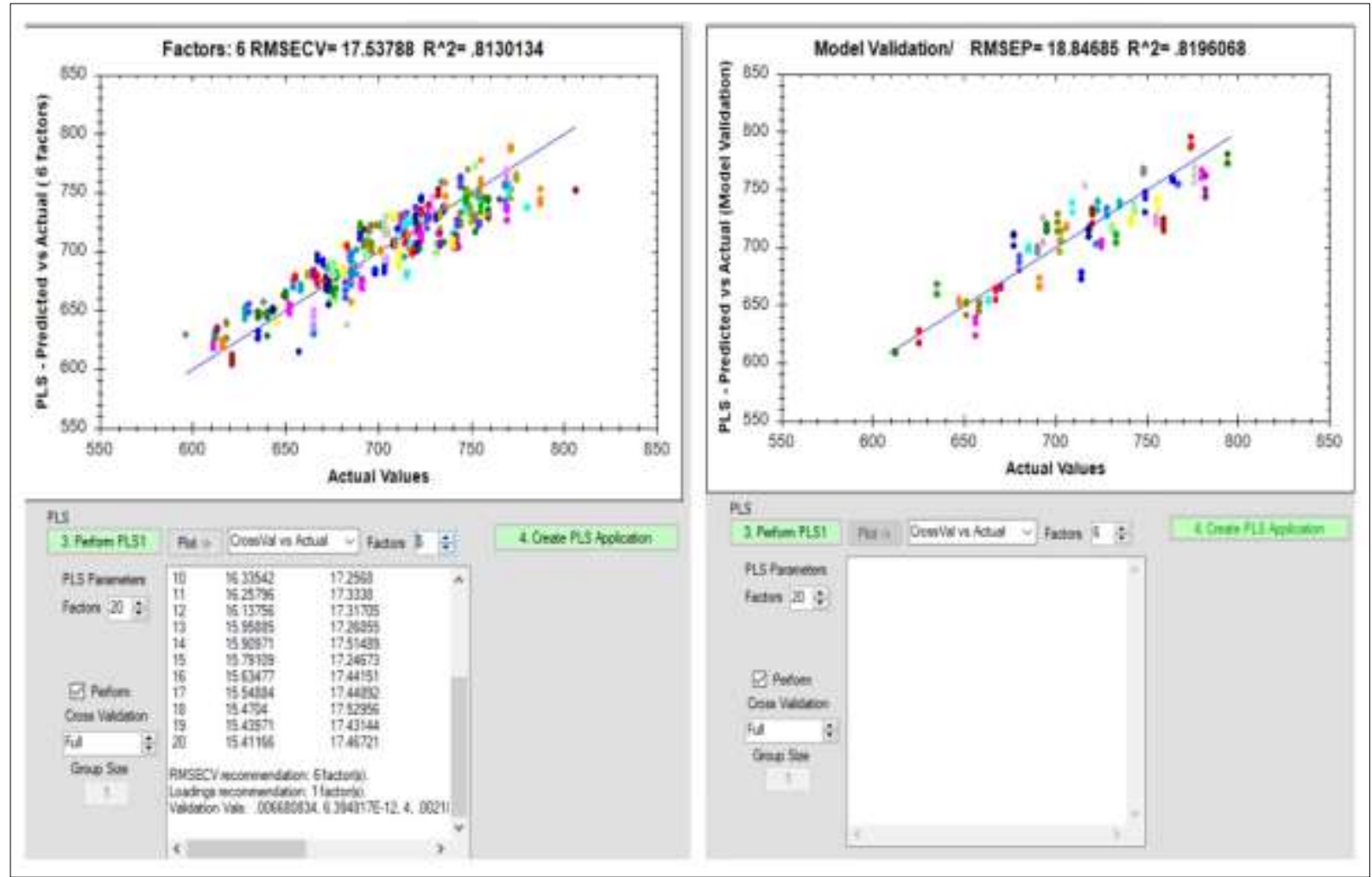

Figura 6. Modelo de Calibración y Validación en el Software Thermo Scientific 
Cuadro 7. Comparación Método TAPPI vs NIR

\begin{tabular}{ccccc}
\hline \multirow{2}{*}{ Método } & Valor Medio & $\begin{array}{c}\text { Desviación } \\
\text { Estándar }\end{array}$ & Valor Mínimo & Valor Máximo \\
\cline { 2 - 5 } & \multicolumn{4}{c}{$\left(\mathbf{k g} / \mathbf{m}^{\mathbf{3}}\right)$} \\
\hline Tappi & 712,94 & 37,08 & 612,15 & 806,27 \\
\hline NIR & 712,53 & 33,59 & 608,96 & 795,85 \\
\hline
\end{tabular}

La media de la densidad básica calculada por NIR es de $712,53 \mathrm{~kg} / \mathrm{m}^{3}$ con una desviación estándar de $33,59 \mathrm{~kg} / \mathrm{m}^{3}$, valores similares a los obtenidos por el método tradicional. El mínimo registrado fue de $608,95 \mathrm{~kg} / \mathrm{m}^{3}$ y el máximo fue de $795,85 \mathrm{~kg} / \mathrm{m}^{3}$.

A partir de estos resultados, se puede concluir que la espectroscopía NIR, es una herramienta capaz de poder estimar la densidad básica de la madera, para árboles de la especie Acacia mearnsii, obteniendo resultados similares entre ambas metodologías.

\section{- Mejora de los Costos}

Al realizar una estimación y clasificación de gastos es posible señalar una disminución en los costos involucrados, donde se observó que el método NIR, necesitó de menos equipos y recursos humanos que el proceso aplicando la metodología Tappi, derivado fundamentalmente de la reducción de actividades en terreno para obtener las rodelas, lo que implica para la Institución menores gastos en recursos humanos y materiales.

\section{Controlar}

Para asegurar que el nuevo proceso mantenga buenos resultados a lo largo del tiempo, se decidió generar un plan de control, el cual debe ser informado a todo el personal involucrado, con la finalidad de generar un protocolo en el control de calidad del nuevo proceso. El plan de control se menciona a continuación.

- Se establece un encargado de evaluar el funcionamiento del proceso de cálculo de densidad básica de la madera mediante espectroscopia NIR

- Este funcionario, cada vez que se quiera saber la densidad básica de la madera de un ensayo, debe medir los tiempos de 30 muestras, agregarlos a las muestras antiguas y revisar el desempeño.

- En caso de que existan puntos atípicos, revisar las causas y realizar un análisis correspondiente

- Cada vez que se requiera realizar este proceso, se debe hacer una capacitación a todo el personal que participe del proyecto.

- Emitir un informe cada vez que se realice el proceso, para llevar un registro histórico de los índices evaluados en las actividades, generando al mismo tiempo una comparación entre una toma de muestra y otra.

- $\quad$ Ingreso de toda la información a bases de datos

\section{ANÁLISIS DE LOS RESULTADOS}

La metodología Seis Sigma fue una herramienta adecuada para la identificación y solución de problemas, debido a que analiza todo el proceso identificando las causas de los problemas y con ello, buscar diferentes alternativas de solución. Además, al utilizar las diferentes herramientas de calidad, se pudo evidenciar distintos indicadores del proceso antiguo y nuevo, permitiendo realizar una comparación entre ambos, y concluir que proceso fue más eficiente. 
Se logró cumplir con el objetivo de proponer un proceso no destructivo, a través de la metodología NIR para la estimación de la densidad básica de la madera, en donde se utilizó un modelo de regresión multivariante de mínimos cuadrados parciales, para la calibración de instrumento. El modelo final tiene un nivel predictivo de calibración de $81,3 \%$, con un RMSECV de $17,54 \mathrm{~kg} / \mathrm{m}^{3}$, además el modelo de validación generó un $\mathrm{R}^{2}$ de $81,96 \%$ y un RMSEP de $18,85 \mathrm{~kg} / \mathrm{m}^{3}$.

El estudio de densidad básica de la madera, para Acacia mearnsii de 13 años de edad, dio como resultado en promedio de $712,94 \mathrm{~kg} / \mathrm{m}^{3}$ con una desviación estándar de $37,08 \mathrm{~kg} / \mathrm{m}^{3}$, para el método tradicional, y de $712,53 \mathrm{~kg} / \mathrm{m}^{3}$ de promedio y de $33,59 \mathrm{~kg} / \mathrm{m}^{3}$ de desviación estándar en el método NIR.

Mediante la metodología Seis Sigma, modelo DMAMC, se pudo identificar las causas del problema, las cuales fueron clasificadas en 4 categorías, donde la de mayor importancia fue la del "Método", con un 45,7\%, seguido del "Clima" con un 24,6\%, "Máquinas" con un 21,7\% y "Personal" con un $8 \%$.

En cuanto a la preparación de las muestras, la diferencia de tiempos entre el método tradicional y el método NIR es de mínimo 20 días, siendo el método tradicional el más largo. Por otra parte, los tiempos de cálculo de densidad de la madera de ambos métodos fueron similares, presentando tiempos promedios de 277 segundos por muestra en el método tradicional y 273 segundos por muestra en el método NIR. El método NIR posee una variabilidad menor, en donde el método tradicional presenta una desviación estándar estimada de 87,98 segundos, mientras que el método NIR presenta una desviación estándar estimada de 21,65 segundos.

En cuanto a los indicadores de calidad, se pudo ver que en el método tradicional el nivel sigma fue de $0,18, C_{p}$ de 0,26 y $C_{p k}$ de 0,23 , generando aproximadamente 440.000 defectos en un millón (DPMO). Los indicadores de calidad del método NIR señalaron un nivel sigma de 3, con un $C_{p}$ de 1,08 y $C_{p k}$ de 1 . Los DPMO calculados fueron de 1.700. El método tradicional generó mayor costo respecto del método utilizando el NIR, derivado fundamentalmente del menor tiempo requerido en el proceso de este último, que significa menores costos en recursos humanos, operación y otros. En el Cuadro 8 se presenta un resumen de los resultados.

Cuadro 8. Resumen de Resultados

\begin{tabular}{|c|c|c|c|c|}
\hline Indicador & Unidad & $\begin{array}{l}\text { Metodología } \\
\text { Actual }\end{array}$ & $\begin{array}{l}\text { Metodología } \\
\text { Propuesta }\end{array}$ & $\begin{array}{c}\text { Variación en } \\
\text { porcentaje }\end{array}$ \\
\hline Promedio densidad de la madera & $\left(\mathrm{kg} / \mathrm{m}^{3}\right)$ & 712,94 & 712,53 & $-0,06 \%$ \\
\hline Desviación estándar densidad & $\left(\mathrm{kg} / \mathrm{m}^{3}\right)$ & 37,08 & 33,59 & $-9,41 \%$ \\
\hline Tiempos preparación de la muestra & (Días) & 20 & 1 & $-95,00 \%$ \\
\hline Tiempos promedio de cálculo de densidad & (Segundos) & 277 & 273 & $-1,44 \%$ \\
\hline $\begin{array}{l}\text { Desviación estándar de los tiempos de cálculo de } \\
\text { densidad }\end{array}$ & $\left(\mathrm{kg} / \mathrm{m}^{3}\right)$ & 87,98 & 21,65 & $-75,39 \%$ \\
\hline Nivel sigma & & 0,18 & 2,99 & $1561,11 \%$ \\
\hline $\mathrm{Cp}$ & & 0,26 (No capaz) & 1,08 (Capaz) & $315,38 \%$ \\
\hline Cpk & & 0,23 (No capaz) & 1 (Capaz) & $334,78 \%$ \\
\hline DPMO & & 440.059 & 1.732 & $-99,61 \%$ \\
\hline
\end{tabular}

Al observar los valores predictivos obtenidos en la calibración y validación del modelo PLS, se puede decir que el modelo generado refleja una adecuada calibración del equipo, debido a que los $\mathrm{R}^{2}$ son mayores al $80 \%$, lo que permite establecer que el equipo estima la densidad básica de la madera de 
Acacia mearnsii con más de un $80 \%$ de exactitud. Además, los errores asociados al modelo, en comparación con otros estudios, son menores, por lo que se consideró que es posible utilizar el equipo NIR para futuros cálculos de densidad básica en la especie.

En cuanto a los valores de densidad básica obtenida, sus valores son cercanos entre ambos métodos. La diferencia de estos promedios fue de $0,41 \mathrm{~kg} / \mathrm{m}^{3}$, que representa un $0,06 \%$ del promedio bajo la norma Tappi. Esta variación rectifica que el nivel de cálculo de la metodología NIR es adecuada para una estimación de este parámetro.

Observando los tiempos de la preparación de la muestra, el tiempo empleado para el proceso bajo la norma Tappi, es prolongado, no así al emplear la metodología del equipo NIR, que en este caso permitió ahorrar casi un mes en tiempo para poder obtener la variable deseada.

Además, los tiempos promedios empleados para el cálculo de densidad básica de la madera, fueron muy similares, pero la variabilidad mostrada en la metodología NIR fue menor. Esto fue debido a que las muestras fueron homogenizadas, generando muestras de tamaños similares. Con esto, se identificó que el proceso para calcular densidad básica de la madera en Acacia mearnsii, mediante el método tradicional según la norma Tappi T258 om-94, genera tiempos prolongados y variables, en donde como solución se decidió utilizar la espectroscopía NIR, que reduce los tiempos de estimación y además son más homogéneos.

Al realizar un análisis en los indicadores de calidad estudiados, los valores del $C_{p}$ y $C_{p k}$ obtenidos de la metodología Tappi, son menores a 0,3, valores debajo de lo esperado para un proceso que funcione correctamente, donde se espera, que estos índices sean mayores a 1. La consecuencia de este índice bajo es que la estimación de densidad básica requerirá de tiempos de trabajos muy variados y generalmente más altos.

Los índices de calidad $C_{p}$ y $C_{p k}$ de la metodología NIR, son mayores o iguales a 1, lo cual significa que el proceso es capaz de generar tiempos en los límites de especificación, pero mejorables, además, es necesario realizar un constante monitoreo, debido a que ante cualquier desplazamiento de la media 0 aumento en la desviación estándar, podría adulterar los niveles $C_{p}$ y $C_{p k}$, generando una disminución de estos, lo que significa que el proceso podría pasar de ser capaz a dejar de serlo.

En cuanto a los costos, se pudo observar que el método tradicional, necesitó de más equipos y recurso humano que el proceso NIR, que, sumado al tiempo, generó notables diferencias en cuanto al costo involucrado.

\section{CONCLUSIONES}

- Existe una alta demanda de biomasa forestal para diferentes usos en el país, por lo que es importante tener información física y química de la materia prima, en donde la densidad básica de la madera es una de las variables físicas más importante, debido a que con este valor se puede estimar el material seco disponible.

- La aplicación de la metodología Seis Sigma para el análisis del proceso de estimación de la densidad básica de la madera, permitió la identificación y solución de problemas, a través de la observación de todo el proceso, identificando las causas de los problemas y buscando diferentes alternativas de solución.

- Se pudo apreciar los beneficios de la metodología DMAMC (Definir, Medir, Analizar, Mejorar y Controlar), ya que exige definir de manera clara los objetivos del proyecto, caracterizando y delegando las actividades necesarias para cumplir con las metas, y así poder planificar de mejor manera el proceso.

- La metodología señala que, para un adecuado proceso, se requiere describir cada actividad, cuáles son los equipos y recursos necesarios para ejecutarlas, identificando las posibles causas que generan los problemas, analizando y cuantificando, mediante el trabajo en equipo. 
- La metodología vela para que en el futuro el proceso continúe con un buen rendimiento, ya que es necesario realizar un protocolo de control, el cual sirve para identificar nuevos problemas, permitiendo realizar nuevas mejoras a este.

- El uso de la espectroscopía NIR, fue un proceso eficiente para obtener la densidad básica de la madera, por lo que se puede concluir que este equipo es apto para poder estimar este valor en árboles de la especie Acacia mearnsii, siendo además un instrumento versátil para la obtención de información, sobre todo para datos químicos, ya que con los mismo espectros recolectados, el instrumento se puede calibrar para brindar información sobre otros parámetros como nivel de taninos, celulosa, lignina, entre otros componentes que poseen los árboles.

- Al utilizar las diferentes herramientas de calidad, se pudo evidenciar distintos indicadores del proceso antiguo (norma Tappi) y nuevo (NIR), permitiendo realizar una comparación entre ambos.

- Se pudo observar que, en términos de tiempo, apoyado con la metodología Seis Sigma, la utilización de la espectroscopía NIR fue mucho más rápida y además menos variable, siendo además un método no destructivo, debido que no hay necesidad de talar árboles para realizar las estimaciones.

- Se recomienda continuar nutriendo la base de datos del equipo NIR, con espectros provenientes de diferentes especies de Acacia, que sean de diferentes edades y zonas, con la finalidad de obtener mayor variabilidad de espectros, mejorando el modelo propuesto, para así obtener estimaciones más certeras.

- El costo del equipo NIR puede ser un obstáculo para no realizar un mayor uso de esta tecnología, a lo que se agrega el no estar normado en Chile su uso como un equipo apto de estimar parámetros físicos y químicos en biomasa.

- Es importante notar, que la metodología Seis Sigma puede ser empleada para el análisis y mejora de distintos procesos o productos relacionados con la actividad forestal, que, debido a la escasa bibliografía existente, puede ser un área interesante a considerar para nuevos estudios o investigaciones.

\section{REFERENCIAS}

Bahamondez, C., Ferrando, M., Martin, M. \& Pinilla, J.C. (1995). Determinación de funciones de volumen y razón de volumen para eucalipto. Instituto Forestal-Fondef. Documento proyecto Conicyt-Fondef 2/33. 16p.

Bailléres, H., Davrieux, F. \& Ham-Pichavant, F. (2002). Near infrared analysis as a tool for rapid screening of some major wood characteristics in a Eucalyptus breeding program. Annals of Forest Science, 59. Pp: 479-490. https://doi.org/10.1051/forest:2002032

Carter, P., Briggs, D., Ross, R. \& Wang, X. (2005). Acoustic testing to enhance western forest values and meet customer wood quality needs. En: Harrington, C., Schoenholtz, S. (Eds). Productivity of Western forests: a forest products focus. Gen. Tech. Rep. PNW-GTR-642. Portland, OR: U.S. Department of Agriculture, Forest Service, Pacific Northwest Research Station. $176 \mathrm{p}$.

Cooper, P., Jeremic, D., Radivojevic, S., Ung, Y. \& Leblon, B. (2011). Potential of near-infrared spectroscopy to characterize wood products. The Role of Sensors in the New Forest Products Industry and Bioeconomy. Canadian Journal of Forest Research, 41(11): 2150-2157. https://doi.org/10.1139/x11-088

Goldratt, E. \& Cox, J. (1992). The Goal: A Process for Ongoing Improvement, Great Barrington: Vt.: North River Press.

Kannegiesser, U. (1990). Apuntes sobre algunas acacias australianas: 1. Acacia mearnsii De Willd. Ciencia \& Investigación Forestal, 4(2): 195-212. https://doi.org/10.52904/0718-4646.1990.141

Kelley, S., Rials, T., Snell, R., Groom, L. \& Sluiter, A. (2004 a). Use of near infrared spectroscopy to measure the chemical and mechanical properties of solid wood. Wood Science and Technology, 38. Pp: 257-276. https://doi.org/10.1007/s00226-003$\underline{0213-5}$

Kelley, S., Rials, T., Groom, L. \& So, G. (2004 b). Use of near infrared spectroscopy to predict the mechanical properties of six softwoods. Holzforschung, 58(3): 252-260. https://doi.org/10.1515/HF.2004.039

Labbé, Rodrigo, Droppelmann, Fernando, Balocchi, Claudio \& Peredo, Miguel. (2013). Variación de la densidad de la madera y rendimiento pulpable en clones de Eucalyptus globulus evaluado con espectroscopia de infrarrojo cercano. Bosque, 34(3): 263-272. https://doi.org/10.4067/S0717-92002013000300002 
Pinilla, J.C. (2000). Descripción y Antecedentes Básicos sobre Acacia dealbata, Acacia melanoxylon y Acacia mearnsii. Revisión bibliográfica. INFOR. Informe Técnico N¹47. Concepción, Chile. 51 p.

Schimleck, L., Jones, P., Peter, G., Daniels, R. \& Clark, A. (2004). Nondestructive estimation of tracheid length from sections of radial wood strips by near infrared spectroscopy. Holzforschung, 58. Pp: 375-381. https://doi.org/10.1515/HF.2004.057

Snee, R. (2001). Dealing with the Achilles' Heel of Six Sigma Initiatives, Quality Progress, Marzo 2001.

So, C.L., Via, B.K., Groom, L.H., Schimleck, L.R., Shupe, T.F., Kelley, S.S. \& Rials, T.G. (2004) Near Infrared Spectroscopy in the Forest Products Industry. Forest Prod J., 54(3): 6-16.

Tsuchikawa, S. \& Kobori, H. (2015). A review of recent application of near infrared spectroscopy to Wood science and technology. Journal of Wood Science, 61. Pp: 213-220. https://doi.org/10.1007/s10086-015-1467-x

Via, B. (2004). Modeling Longleaf Pine (Pinus palustris Mill.) wood properties using near infrared spectroscopy. A Dissertation Submitted to the Graduate Faculty of the Louisiana State University and Agriculture and Mechanical College. In: Partial Fulfillment of the Requirements for the degree of Doctor of Philosophy. 